\title{
ROAD PROFILE IDENTIFICATION USING ESTIMATION TECHNIQUES: COMPARISON BETWEEN INDEPENDENT COMPONENT ANALYSIS AND KALMAN FILTER
}

\author{
Mariem Miladi Chaabane, Dorra Ben Hassen, Mohamed Slim Abbes \\ Mechanics, Modeling and Production Laboratory, National Engineering School of Sfax (ENIS), Sfax, Tunisia \\ e-mail: dorra.benhassen@yahoo.fr \\ Selahattin Caglar Baslamisli \\ Hacettepe University, Department. of Mechanical Engineering, Beytepe, Ankara, Turkey \\ FAKHer ChaAri, Mohamed HAdDar \\ Mechanics, Modeling and Production Laboratory, National Engineering School of Sfax (ENIS), Sfax, Tunisia
}

\begin{abstract}
This paper focuses on the identification of a road profile disturbance acting on vehicles. Vehicles are subjected to many kinds of excitation sources such as road profile irregularities, which constitute a major area of interest when designing suspension systems. Indeed, determining the road profile is important for passive suspension design on the one hand and for determining an appropriate control law for active suspensions on the other. Direct measurement techniques of the road profile are expensive, so solutions based on estimation theory are needed. The aim of this paper is to characterize the road excitation using the Independent Component Analysis (ICA). This proposed method can reconstruct original excitation sources by using physically measurable signals of the system under study. Here, the estimation of road disturbances is considered as output sources and identified from dynamic responses of the vehicle. These responses can be measured via sensors or can be numerically computed. In our case, they are numerically simulated using the Newmark method and consider different types of road profiles. The obtained results are validated after using a comparison with the Kalman filtering. The robustness of the ICA is confirmed via parametric study.
\end{abstract}

Keywords: road excitation, ICA, Kalman filtering, identification techniques, vehicle model

\section{Introduction}

In order to link the vehicle chassis and its wheels to the ground, a suspension composed of springs and dampers is used (Basha and Rao, 2012; Agharkakli et al., 2012). This mechanism aims mainly at isolating the vehicle from road disturbances and providing passenger comfort ( $\mathrm{Li}$ et al., 2014; Purushotham, 2013). In the past decades, many researchers focused on improving road handling and safety. Some of them proposed to use active control for these purposes. A method based on active damping control was firstly developed by Karnopp (1983) and Karnopp and Heess (1991). Others provided alternative algorithms (Gopala Rao and Narayanan, 2008; Saveresi and Spelta, 2009). In this context, Fang et al., 2011) used a double loop control in order to study a dynamic model of a semi-active suspension. This model showed effective results to isolate external vibrations. Recently, Unger et al. (2013) used a new modified road model to develop semi-active suspension control. He showed that this model could successfully be implemented in a real vehicle in order to compensate the road disturbance.

Other works dealt with the study of the influence of the road excitation on the suspension performance. For example, Hunt (1991) examined the dynamic response of vehicles subjected 
to a random excitation due to the road profile. Also Bayrakdar (2010) studied the dynamic response of the vehicle subjected to a harmonic excitation using single and multi-degree of freedom systems. Therefore, the knowledge of the road profile is very important since it has a big impact on both the passengers comfort and ride quality (Yan, 2012). This topic has been studied by many researchers for many decades. Some of them recommended measuring road roughness by visual inspections (Kim et al., 2002). Others proposed the estimation theory to identify the road profile such as Harris et al., 2010) who used the Monte Carlo estimation and Solhmirzaei et al. (2012) who proposed the use of a multi-input multi-output feed forward wavelet neural network. However, these methods have some restrictions. One of the most used techniques nowadays is the Kalman filtering. This method was modified by Fauriat et al. (2016) who used the "Augmented Kalman filter" and showed that this technique gave a good estimation result. But it still has some limitations.

In this paper, extraction of external excitations to which the quarter car model is subjected is done by applying the Independent Component Analysis (ICA). The ICA is based on recovering unknown source signals from the observed signals. Several works used this technique to diagnose mechanical systems in the context of conditional monitoring (Sika, 2008; Gelle et al., 2003). ICA aims to decompose measured signals into a linear combination of independent signals in order to have the measured signals as independent as possible. Akrout et al. (2012) used this technique for the estimation of excitation forces applied to a continuous system and proved its efficiency in reconstructing original signals. The aim of this paper is to investigate the efficiency of applying the ICA technique in identification of external excitations applied to vehicles (Zarzoso and Comon, 2010; Nakamura et al., 2012; Ben Hassen et al., 2017a,c,d). The results of estimation were validated through comparing the obtained results with those of the Kalman filtering developed by Fauriat et al. (2016).

This paper is organized as follows: firstly, description of the studied model and road profile disturbance is done. Then, techniques of estimation are presented: the proposed ICA and validation technique: Kalman filtering. Finally, the reconstructed excitations by the ICA are compared to the original ones and to those obtained by the Kalman filtering. The efficiency of the ICA method is also discussed by studying the influence of the sprung mass variation and the vehicle speed in the estimation process.

\section{Vehicle model and road disturbance}

To study the dynamic behavior of the vehicle, different models of vehicles and road profiles are used.

There are mainly three types of vehicle models: the full car model which comprises vertical displacement of the wheels and body rotations about longitudinal and lateral axis, the half-vehicle model which takes into account pitch or roll motion and finally, the quarter-vehicle model, the simplest one, describing only vertical motions but containing the basic characteristics of the full model.

The road distribution can be modeled as continuous vibrations or singular perturbations. Singular perturbations are defined as a high shock amplitude that occurs on a smooth road while continuous vibrations are modeled as random excitations due to a rough road.

In this paper, a quarter car model subjected to a random excitation is studied.

\subsection{Studied vehicle model: quarter car model}

A quarter car model with two degrees of freedom is studied in this paper (Fig. 1). $X_{1}$ is the displacement of the sprung mass $m_{1}$ while $X_{2}$ is the displacement of the unsprung mass $m_{2}$. 


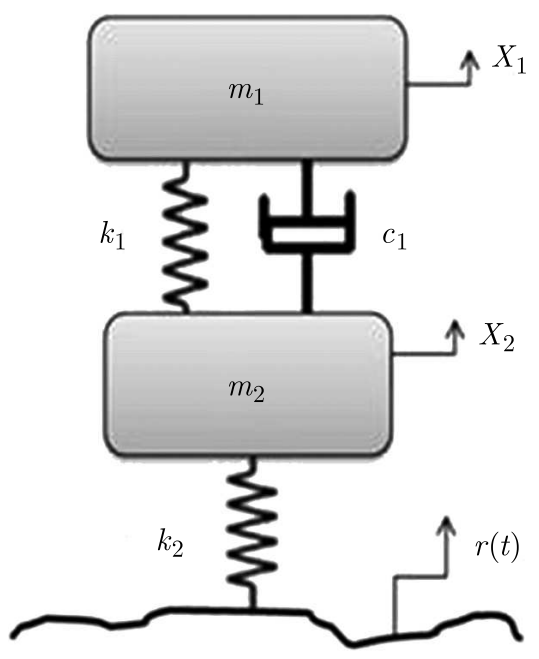

Fig. 1. Quarter car model

The equations of motion for the system at hand may be written as follows

$$
\begin{aligned}
& m_{1} \ddot{X}_{1}+k_{1}\left(X_{1}-X_{2}\right)+c_{1}\left(\dot{X}_{1}-\dot{X}_{2}\right)=0 \\
& m_{2} \ddot{X}_{2}+k_{1}\left(X_{2}-X_{1}\right)+c_{1}\left(\dot{X}_{2}-\dot{X}_{1}\right)+k_{2}\left(X_{2}-r(t)\right)=0
\end{aligned}
$$

In the matrix form, these equations can be written as

$$
\mathbf{M} \ddot{\mathbf{X}}+\mathbf{C} \dot{\mathbf{X}}+\mathbf{K X}=\mathbf{F}
$$

where $\mathbf{X}, \dot{\mathbf{X}}$ and $\ddot{\mathbf{X}}$ are respectively the displacement, velocity and acceleration vectors. $\mathbf{M}, \mathbf{C}$ and $\mathbf{K}$ are respectively the mass, damping and stiffness matrices of the studied system. $\mathbf{F}$ is the excitation force due to the road disturbance $r(t)$.

The matrices that appear in equation (2.2) are given by

$$
\begin{array}{lr}
\mathbf{M}=\left[\begin{array}{cc}
M_{1} & 0 \\
0 & M_{2}
\end{array}\right] & \mathbf{C}=\left[\begin{array}{cc}
c_{1} & -c_{1} \\
-c_{1} & c_{1}
\end{array}\right] \\
\mathbf{K}=\left[\begin{array}{cc}
k_{1} & -k_{1} \\
-k_{1} & k_{1}+k_{2}
\end{array}\right] & \mathbf{F}=\left[\begin{array}{c}
0 \\
k_{2} r(t)
\end{array}\right]
\end{array}
$$

The parameters considered for the suspension system are taken according to the model of Fauriat et al. (2016). They are presented in Table 1.

Table 1. Parameters of the suspension system (Fauriat et al., 2016)

\begin{tabular}{|l|c|c|}
\hline \multicolumn{1}{|c|}{ Parameter } & Value & Unit \\
\hline \hline Sprung mass & $m_{1}=372$ & $\mathrm{~kg}$ \\
\hline Unsprung mass & $m_{2}=59$ & $\mathrm{~kg}$ \\
\hline Suspension stiffness & $k_{1}=36540$ & $\mathrm{~N} / \mathrm{m}$ \\
\hline Tire stiffness & $k_{2}=242000$ & $\mathrm{~N} / \mathrm{m}$ \\
\hline Suspension damping & $c_{1}=3300$ & $\mathrm{Ns} / \mathrm{m}$ \\
\hline
\end{tabular}

\subsection{Road disturbance: random road profile}

Concerning the external excitation due to the road disturbance, a random road profile is considered in this study. This profile is constructed according to ISO 8608 (1995) (Table 2) 
Table 2. Road profile classification

\begin{tabular}{|c|c|c|c|}
\hline \multirow{2}{*}{$\begin{array}{c}\text { Road } \\
\text { class }\end{array}$} & \multicolumn{3}{|c|}{ Degree of roughness $G_{d}\left(n_{0}\right)\left[10^{-6} \mathrm{~m}^{3}\right]$} \\
\cline { 2 - 4 } & Lower limit & Geometric mean & Upper limit \\
\hline \hline Road A & - & 16 & 32 \\
\hline Road B & 32 & 64 & 128 \\
\hline Road C & 128 & 256 & 512 \\
\hline Road D & 512 & 1024 & 2048 \\
\hline Road E & 2048 & 4096 & 8192 \\
\hline
\end{tabular}

which classifies profiles to different categories based on the power spectral density (PSD) (Yan, 2012).

The general form of the PSD is

$$
G_{d}=G_{d}\left(n_{0}\right)\left(\frac{n}{n_{0}}\right)^{-w}
$$

where $n_{0}\left(n_{0}=0.1\right.$ cycle $\left./ \mathrm{m}\right)$ is the reference spatial frequency, $n$ is the spatial frequency, $w$ is the exponent of the PSD and $G_{d}\left(n_{0}\right)$ is the displacement PSD. The classification of different profiles is defined by Table 2 .

The construction of the road roughness is done by the method of the Integral White Noise Model (Yan, 2012) which assumes that the road roughness is a result of the filtered white noise. It is defined by the following equation

$$
\dot{q}(t)=2 \pi n_{0} w_{1}(t) \sqrt{G_{d}\left(n_{0}\right) v}
$$

where $w_{1}(t)$ is the Gaussian white noise with a variance equal to $1, q(t)$ is the road roughness and $v$ is the vehicle velocity.

\section{Estimation techniques}

As mentioned previously, the road profile affects both the vehicle dynamics and the passengers comfort. Therefore, a precise knowledge of the imperfection of the road is essential. Thus, several researches have been estimating the road profile. Some of the results are based on direct measurements (Kim et al., 2002) of the road profiles, whereas others on numerical methods (Solhmirzaei et al., 2012).

In this paper, two techniques of estimation are studied: the Independent Component Analysis (Ben Hassen et al., 2017d; Taktak et al., 2012; Abbes et al., 2011), which is the proposed method to estimate the road profile and the Kalman filter (Fauriat et al., 2016), which is used to validate the ICA.

\subsection{The proposed algorithm: Independent Component Analysis}

The vector $\mathbf{O}$ of the observed signals is written as (Welch and Bishop, 2006; Kalman, 1960)

$$
\mathbf{O}=\mathbf{M}_{I C A} \mathbf{S}
$$

where $\mathbf{O}$ is the vector of the observed signals (in this paper, the vector of the observed signals represents the sprung mass acceleration), $\mathbf{M}_{I C A}$ - the mixing matrix, $\mathbf{S}$ - the vector of source signals (in this paper, the vector of the source signals represents the estimated road profile).

The task consists of estimating both $\mathbf{M}_{I C A}$ and $\mathbf{S}$ by knowing only the vector $\mathbf{O}$. This estimation must be done under some assumptions given in (Hyvärinen and Oja, 2000) as follows: 
- The components of the vector $\mathbf{S}$ are statistically independent.

- The number of the observed signals is equal to the number of the estimated sources.

- The components of the vector $\mathbf{S}$ must have a non-Gaussian distribution.

Taking into account these general assumptions, the ICA can define each column of the matrix $\mathbf{M}_{I C A}$ and then compute the separating matrix $\mathbf{W}$ as the inverse of the matrix $\mathbf{M}_{I C A}$.

Then the ICA estimates the corresponding source signal defined by

$$
\mathbf{S}=\mathbf{W O}
$$

Note that $\mathbf{W}$ must satisfy the criterion of a non-Gaussian distribution, so it must maximize the kurtosis (Ben Hassen et al., 2017d).

In order to have a successful extraction of the estimated sources and the mixing matrix, the observed signal $\mathbf{O}$ undergoes some pre-treatments (Hyvärinen and Oja, 2000). In fact, these measured signals must be centered and whitened in order to simplify the ICA algorithm.

After validating all the pre-treatments, the recovered signal can be separated. So we are able to determine each column of the separating matrix by the ICA, and then the source related to this column is extracted. It is defined by

$$
\mathbf{Y}=\mathbf{W}^{\mathrm{T}} \mathbf{O}
$$

Finally, after determination of the first column of the matrix $\mathbf{W}$, the ICA uses the deflation approach to extract the estimated sources. So, each source will be chosen once with a multiplying factor.

Figure 2 summarizes the concept of the ICA algorithm (Abbes et al., 2011; Taktak et al., 2012).

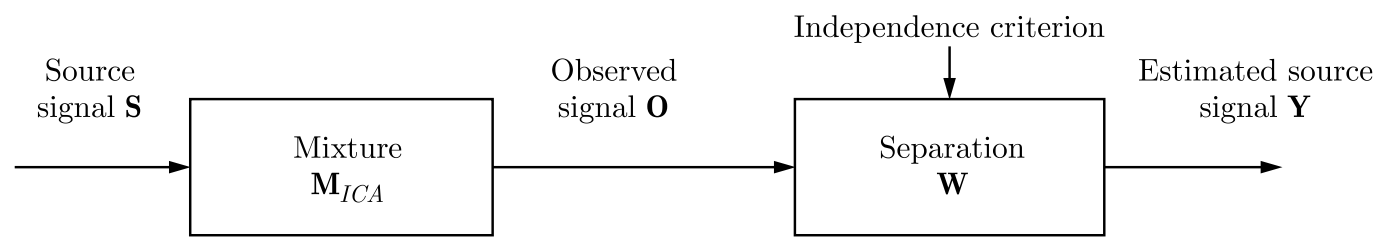

Fig. 2. ICA concept

In this study, the vector of the observed signal $X$ is constructed only by the sprung mass acceleration, Eq. (2.1), so

$$
\mathbf{O}(t)=\ddot{\mathbf{X}}_{1}
$$

\subsection{Augmented Kalman filtering}

The Kalman filter is a predictor-corrector type estimator (Kalman, 1960; Welch and Bishop, 2006). It incorporates all information that can be provided to it in order to estimate the current value of the variables of interest (Fauriat et al., 2016). This technique is used as real-time estimation in the context of suspension control.

In this study, application of the augmented Kalman filter to the quarter-vehicle model is used in order to estimate the road profile. Compared with the regular Kalman filter, the augmented Kalman filter takes into account the excitation of the road profile (Fauriat et al., 2016) as mentioned in Eq. (3.5). This leads to the best control of the quality of the obtained estimation results (Fauriat et al., 2016).

The quarter car model is considered linear, continuous and invariant in time, so it can be described by a representation of the following state space

$$
\dot{\mathbf{x}}(t)=\mathbf{A x}(t)+\mathbf{B} u(t) \quad \text { bf } y\left(t_{i}\right)=\mathbf{C x}\left(t_{i}\right)+\mathbf{D} u\left(t_{i}\right)
$$


where

$$
\begin{array}{rlrc}
\mathbf{A}=\left[\begin{array}{cccc}
-\frac{c_{1}}{M_{1}} & \frac{c_{1}}{M_{1}} & -\frac{k_{1}}{M_{1}} & \frac{k_{1}}{M_{1}} \\
\frac{c_{1}}{M_{2}} & -\frac{c_{1}}{M_{2}} & \frac{k_{1}}{M_{2}} & -\frac{k_{1}+k_{2}}{M_{2}} \\
0 & 0 & 1 & 0 \\
0 & 0 & 0 & 1
\end{array}\right] & \mathbf{B}=\left[\begin{array}{c}
0 \\
\frac{k_{2}}{M_{2}} \\
0 \\
0
\end{array}\right] \\
\mathbf{C}=\left[\begin{array}{cccc}
0 & 0 & 1 & -1 \\
-\frac{c_{1}}{M_{1}} & \frac{c_{1}}{M_{1}} & -\frac{k_{1}}{M_{1}} & \frac{k_{1}}{M_{1}} \\
\frac{c_{1}}{M_{2}} & -\frac{c_{1}}{M_{2}} & \frac{k_{1}}{M_{2}} & -\frac{k_{1}+k_{2}}{M_{2}}
\end{array}\right] & \mathbf{D}=\left[\begin{array}{c}
0 \\
0 \\
0 \\
\frac{k_{2}}{M_{2}}
\end{array}\right]
\end{array}
$$

and $\mathbf{x}(t)=\left[\ddot{X}_{1}, \ddot{X}_{2}, \dot{X}_{1}, \dot{X}_{2}\right]^{\mathrm{T}}$ is the state vector while the output vector which constitutes the observed signal for the Kalman filter is defined by: $\mathbf{Y}=\left[X_{1}-X_{2}, X_{1}, \ddot{X}_{1}, \ddot{X}_{2}\right]$.

At the first step, discretization of the state system, Eqs. (3.4), is done, so the discrete state matrices are obtained as: $A_{d}=\mathrm{e}^{A d t}$ and $B_{d}=\left(\mathrm{e}^{A d t}-I\right) \mathbf{A}^{-1} \mathbf{B}$. Then by using the augmented state vector $x_{k}^{a}=\left[x_{k}, u_{k}\right]^{\mathrm{T}}$ and the augmented matrix $\mathbf{A}_{a}$ and $\mathbf{C}_{a}$, the discrete time augmented Kalman filter is obtained as

$$
\begin{aligned}
& \mathbf{x}_{k+1}^{a}=\left[\begin{array}{l}
x_{k+1} \\
u_{k+1}
\end{array}\right]=\left[\begin{array}{cc}
A_{d} & B_{d} \\
0 & I
\end{array}\right]\left[\begin{array}{l}
x_{k} \\
u_{k}
\end{array}\right]+\left[\begin{array}{l}
w_{k} \\
\eta_{k}
\end{array}\right]=\mathbf{A}_{a} \mathbf{x}_{k}^{a}+\boldsymbol{\varsigma}_{k} \\
& y_{k}=[C, D]\left[\begin{array}{l}
x_{k} \\
u_{k}
\end{array}\right]+v_{k}=\mathbf{C}_{a} \mathbf{x}_{k}^{a}+v_{k}
\end{aligned}
$$

where $\varsigma_{k}=\left[w_{k}, \eta_{k}\right]^{\mathrm{T}}$ contains the state and excitation perturbations, respectively, while $\mathbf{v}_{k}$ is the measurement perturbation.

The augmented Kalman filter is applied to obtain the equations for two steps:

— the prediction step

$$
\widetilde{\mathbf{x}}_{k+1 / k}^{a}=\mathbf{A}_{a} \widetilde{\mathbf{x}}_{k / k}^{a} \quad P_{k+1 / k}=\mathbf{A}_{a} \mathbf{P}_{k / k} \mathbf{A}_{a}^{\mathrm{T}}+\mathbf{Q}_{a}
$$

— the correction step

$$
\begin{aligned}
& \mathbf{M}_{k+1}=\mathbf{P}_{k+1 / k} \mathbf{C}_{a}^{\mathrm{T}}\left(\mathbf{C}_{a} \mathbf{P}_{k+1 / k} \mathbf{C}_{a}^{\mathrm{T}}+\mathbf{R}\right)^{-1} \\
& \widetilde{\mathbf{x}}_{k+1 / k+1}^{a}=\widetilde{\mathbf{x}}_{k+1 / k}^{a}+\mathbf{M}_{k+1}\left(\mathbf{y}_{k+1}-\mathbf{C}_{a} \widetilde{\mathbf{x}}_{k+1 / k}^{a}\right) \\
& \mathbf{P}_{k+1 / k+1}=\mathbf{P}_{k+1 / k}-\mathbf{M}_{k+1} \mathbf{C}_{a} \mathbf{P}_{k+1 / k}
\end{aligned}
$$

where $\mathbf{P}_{k+1}$ is the prediction matrix and $\mathbf{M}_{k+1}$ is the correction matrix.

The tuning parameters of the Kalman filtering theory are constructed by the covariance matrices $\mathbf{Q}$ and $\mathbf{R}$ (Fauriat et al., 2016). They are chosen via empirical assessment derived from the tuning operation (Fauriat et al., 2016). They are constant in this study

$$
\mathbf{Q}=\left[\begin{array}{ccccc}
10^{-4} & 0 & 0 & 0 & 0 \\
0 & 10^{-2} & 0 & 0 & 0 \\
0 & 0 & 10^{-8} & 0 & 0 \\
0 & 0 & 0 & 10^{-7} & 0 \\
0 & 0 & 0 & 0 & 10^{-7}
\end{array}\right] \quad \mathbf{R}=\left[\begin{array}{cccc}
10^{-6} & 0 & 0 & 0 \\
0 & 10^{-6} & 0 & 0 \\
0 & 0 & 1 & 0 \\
0 & 0 & 0 & 100
\end{array}\right]
$$




\section{Numerical results and discussions}

This Section describes the results of simulation for the quarter-car suspension system with the ICA. The obtained results are compared with those of the augmented Kalman filter to observe their performance. This comparison is done with the computation of the RMS of the error between the true profile and the estimated one

$$
R M S=\sqrt{\frac{1}{T_{2}}-T_{1} \int_{T 1}^{T 2}\left(y_{1}-y_{2}\right)^{2}} d t
$$

where $y_{1}$ represents the real road profile $r(t)$, Eq. (2.1), and $y_{2}$ the estimated profile $S(t)$, Eq. (3.2).

Then, a brief parametric study is done to investigate the capabilities and limitations of the two studied methods.

\subsection{Random profile: road type $\mathbf{A}$}

Starting from the acceleration of the sprung mass, the ICA method is used in order to extract the estimated road profile. In the Kalman theory, the observed signal is constructed by the suspension deflection, sprung mass displacement and the acceleration of the sprung and unsprung mass. A random profile of type A is being estimated by the two methods for a vehicle speed equal to $54 \mathrm{~km} / \mathrm{h}$. The obtained results are shown in Figs. 3 and 4.

(a)

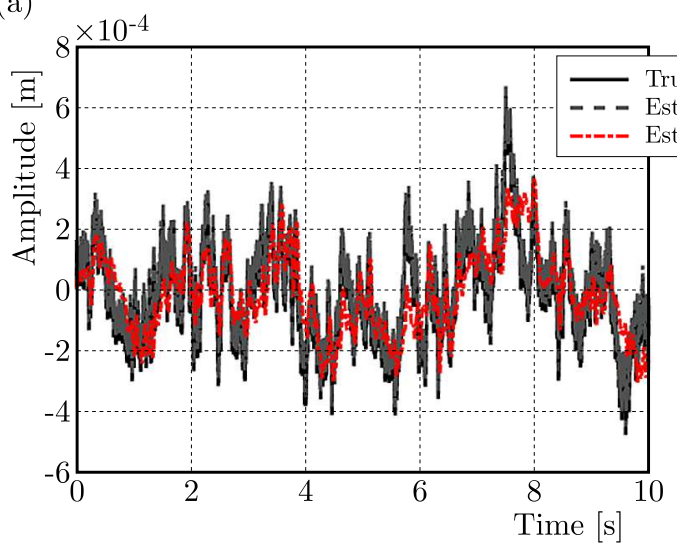

(b)

$$
4 \stackrel{\times 10^{-4}}{\check{r}}
$$

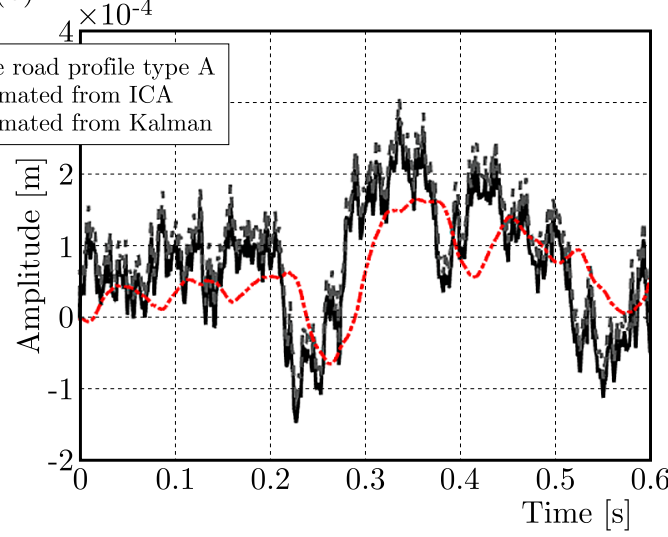

Fig. 3. Road type A: comparison between the true and the estimated profiles (left). Zoomed-in comparison (right)

The results displayed in Figs. 3 and 4 show that both the ICA and the Kalman filtering theory can estimate the true road profile. However, it is noticed that in the time-domain the ICA gives a more precise result than the Kalman theory, which presents a small lag as mentioned by the RMS of the relative error between the true profile and the estimated one presented in Table 3.

Table 3. RMS of the relative error between the true profile (road type A) and the estimated one

\begin{tabular}{|l|c|}
\hline & RMS [m] \\
\hline \hline ICA algorithm & 0.0094 \\
\hline Kalman algorithm & 0.14 \\
\hline
\end{tabular}




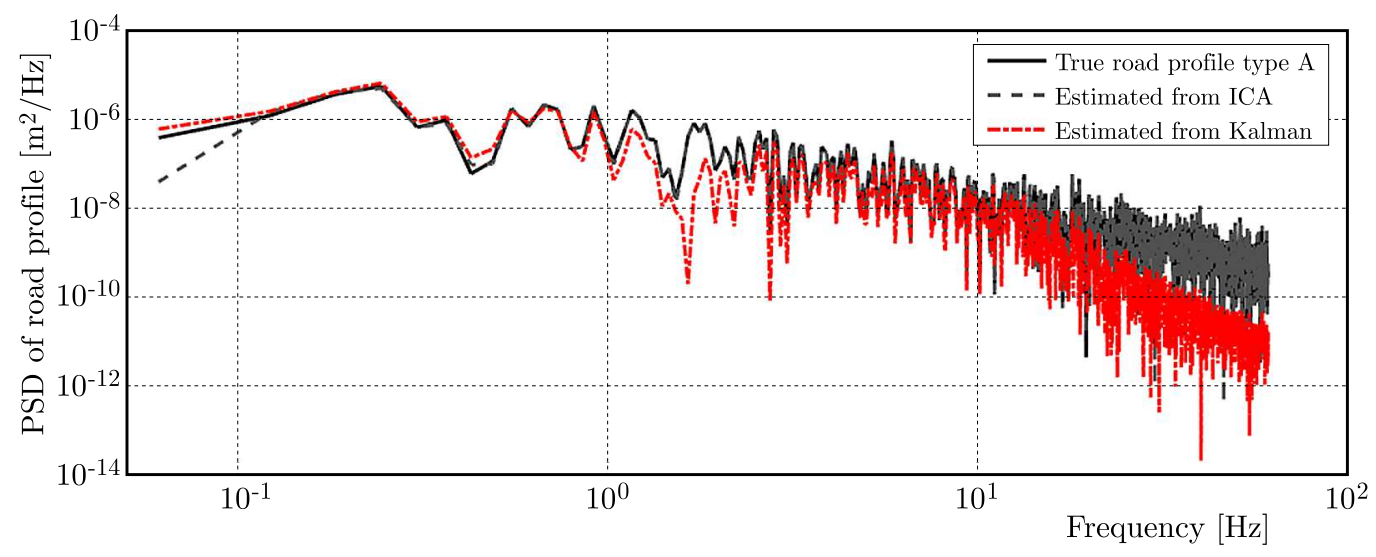

Fig. 4. Road type A: comparison between the estimate and the true profiles in terms of PSDs

In the PSD spectrum, it is clear that there is a loss of content on the high frequency band when the Kalman method is used. According to Fauriat et al. (2016), this phenomenon can not be avoided. Nevertheless, there is a good agreement between the real profile and the estimated with the ICA. A short discrepancy in the low-band frequency is obtained but it does not affect the vehicle response (Fauriat et al., 2016).

\subsection{Composed random profile}

In this part, the road disturbance is constructed with a series of random profiles which are: road A-road C-road E. The use of this type of profile is done to check if the two studied methods are able to estimate a combined road profile.

The obtained results are presented by the following Figs. 5 and 6 .

(a)

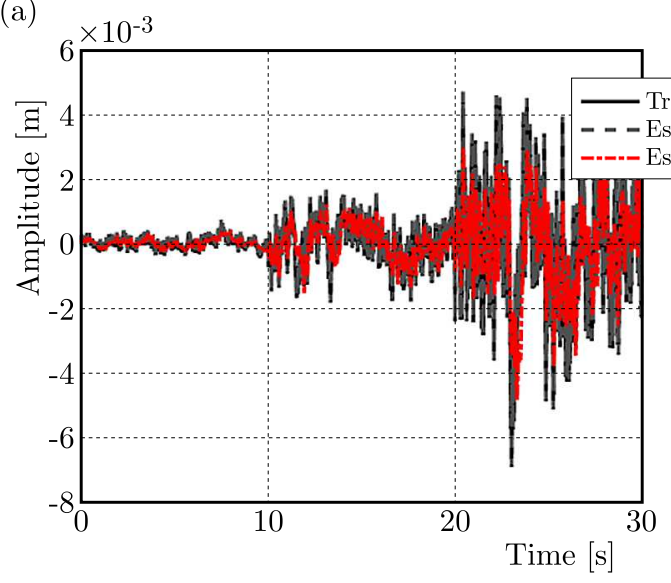

(b)

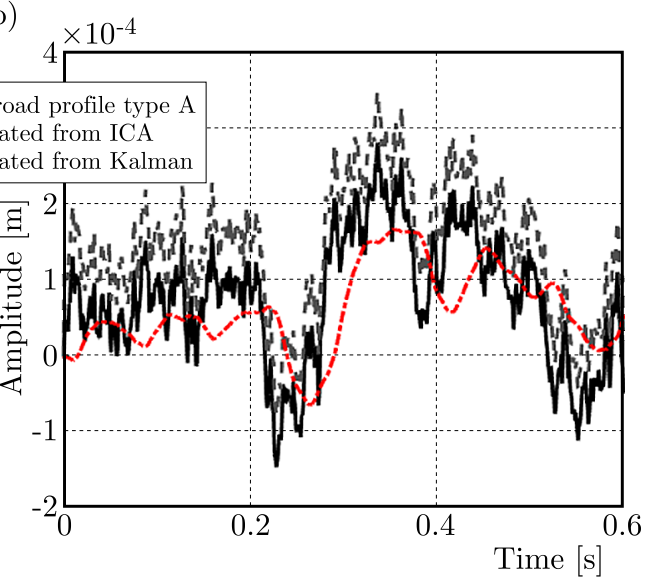

Fig. 5. Composed random profile: comparison between the true and the estimated profiles (left).

Zoomed-in comparison (right)

When observing Figs. 5 and 6, it can be said that both used methods can estimate the road profile even if it is constructed by a combination of different profile types. As in the previous case, the results obtained by the ICA remain more accurate both in the time and frequency domain, and this is proved by Table 4 . 


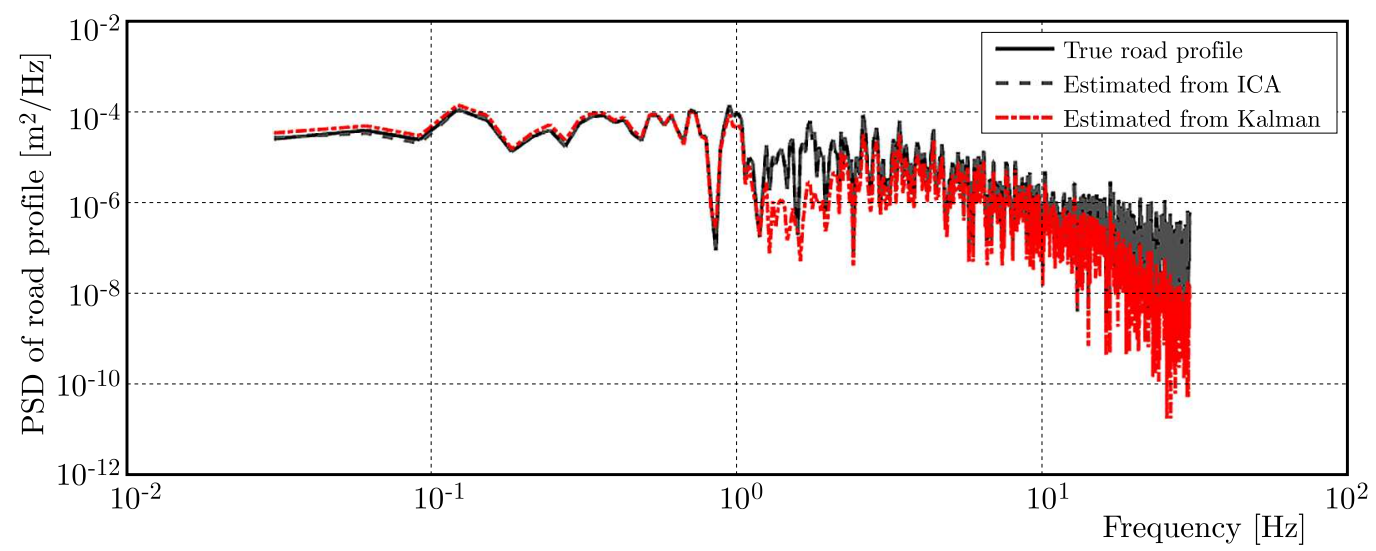

Fig. 6. Composed random profile: comparison between the estimate and the true profiles in terms of PSDs

Table 4. RMS of the relative error between the true profile: composed random profile and the estimated one

\begin{tabular}{|l|c|}
\hline & RMS [m] \\
\hline \hline ICA algorithm & 0.001 \\
\hline Kalman algorithm & 0.18 \\
\hline
\end{tabular}

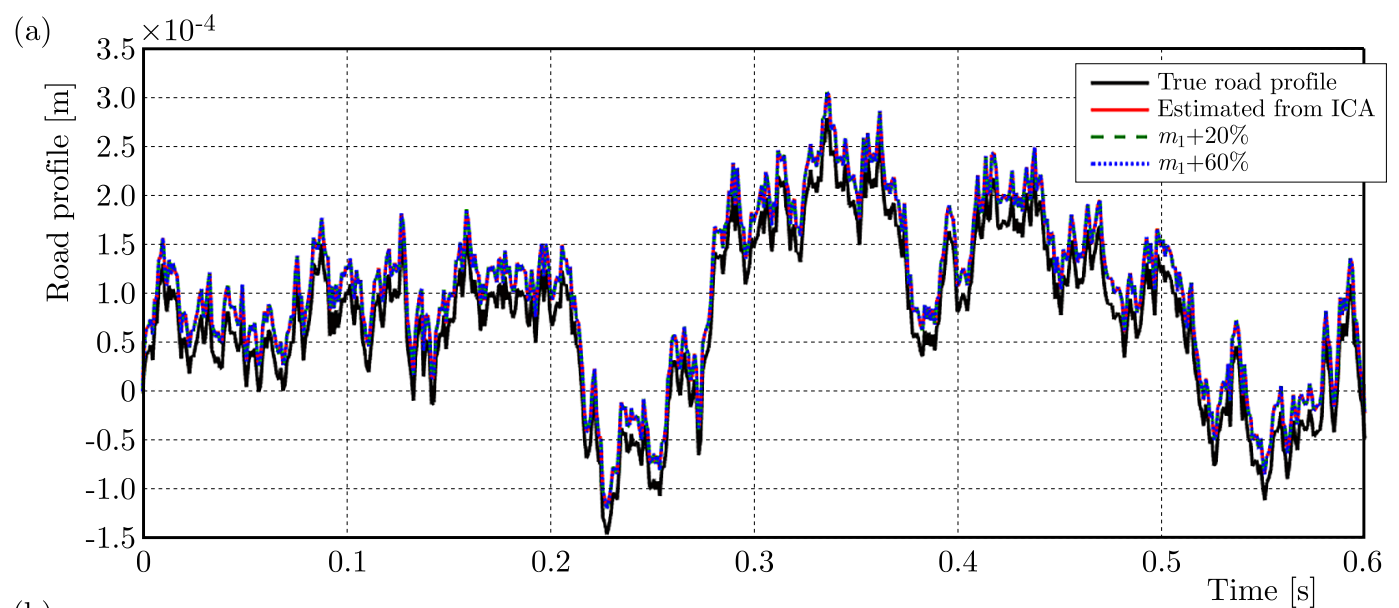

(b)

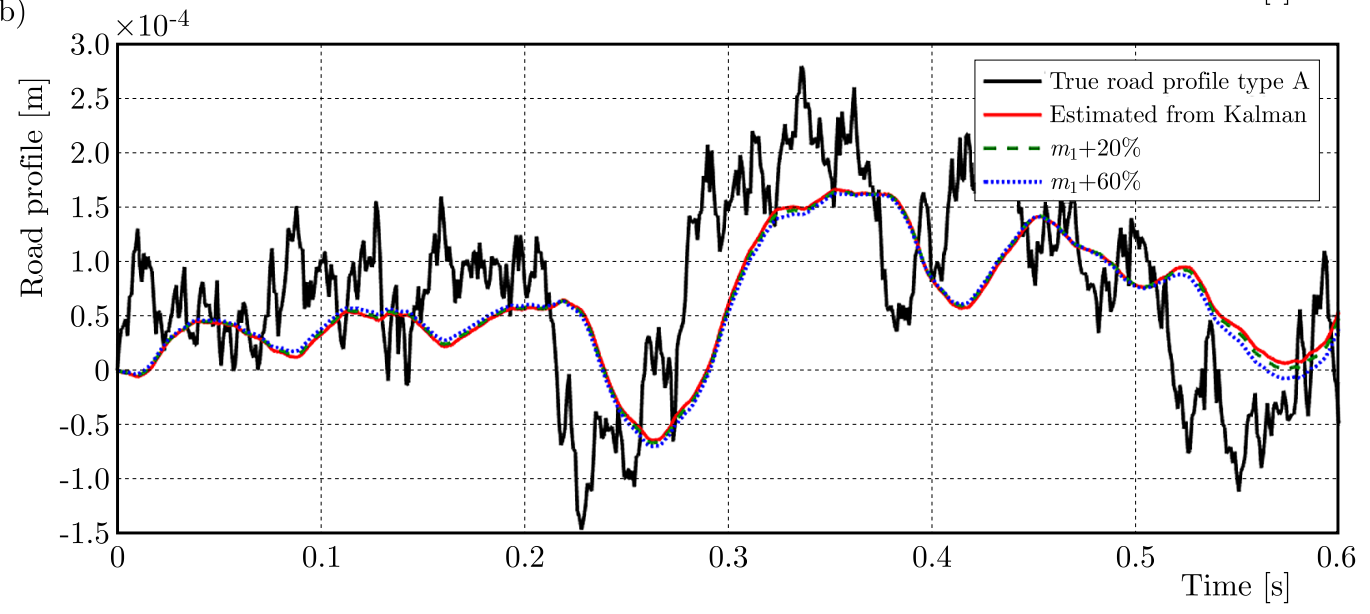

Fig. 7. Effect of variation of sprung mass on the estimation process: (a) ICA algorithm,

(b) Kalman filtering 


\subsection{Parametric study}

\subsubsection{Sprung mass variation}

Since any vehicle whose major activity is transport of people or items, we propose in this part to study the efficiency of the ICA algorithm and the Kalman filtering method via variation of the sprung mass. Hence, we assume that an additional payload is applied to the sprung mass by a range of $20 \%$ and $60 \%$ as shown in Fig. 7 . The presented results are computed for a constant vehicle speed equal to $54 \mathrm{~km} / \mathrm{h}$.

In this figure, it is observed that the ICA produced identical estimation of the road profile with increasing mass. However, the Kalman filtering is a little sensitive to the sprung mass variation.

\subsubsection{Vehicle speed variation}

In this part, we are going to test the vehicle speed variation on the estimation techniques. A speed of $30 \mathrm{~km} / \mathrm{h}$ and $110 \mathrm{~km} / \mathrm{h}$ is applied. The results of the estimation are shown in the following Figs. 8 and 9.

(a)

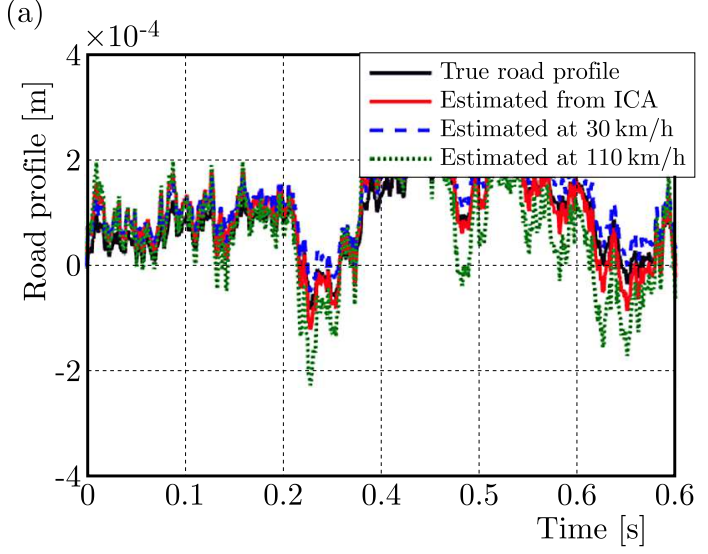

(b)

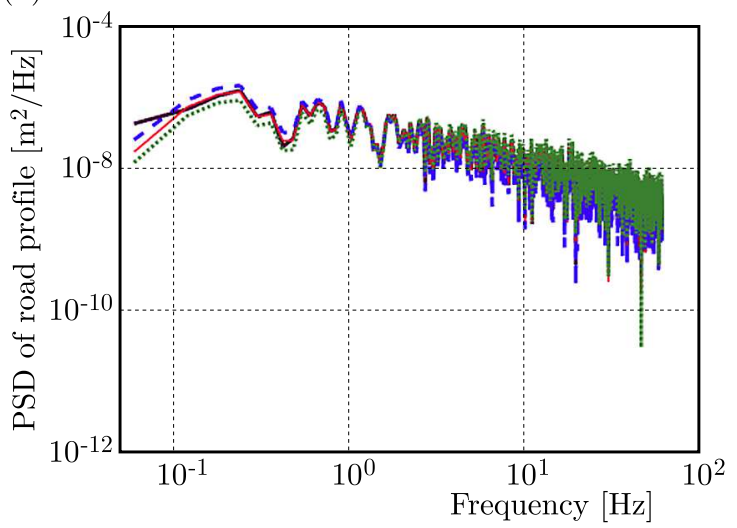

Fig. 8. Effect of variation of the vehicle speed on the estimation by the ICA. Estimated road profiles and the real road profile (left). PSD of different estimates and the real road profile (right)

(a)

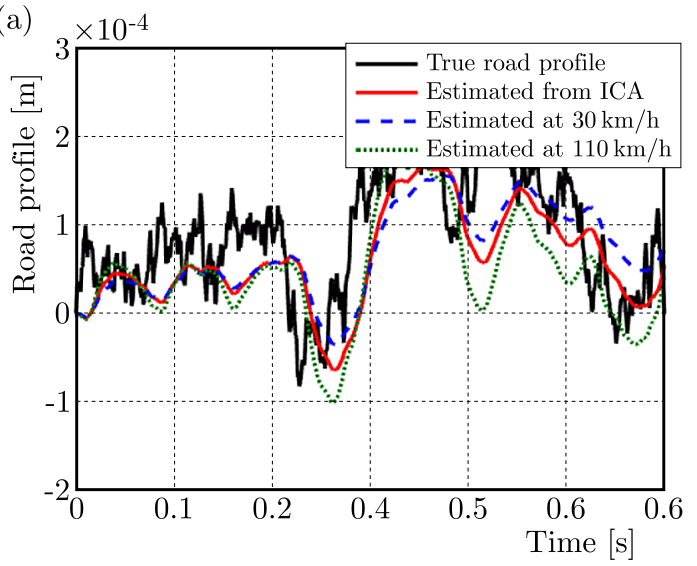

(b)

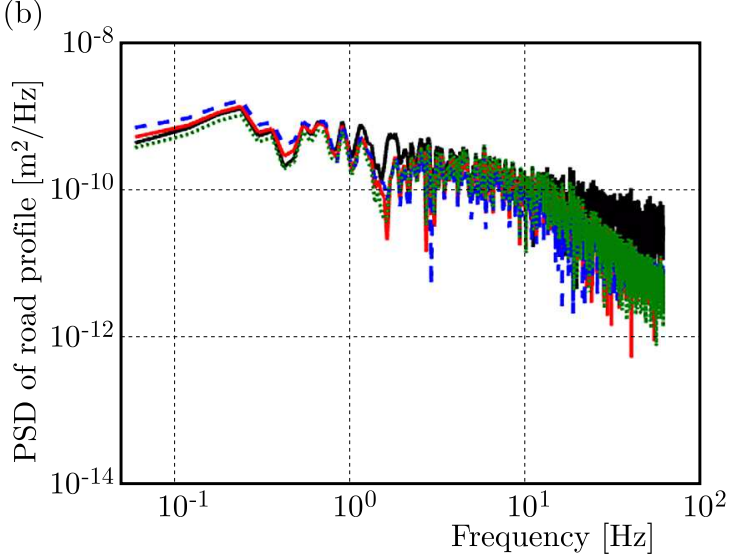

Fig. 9. Effect of variation of the vehicle speed on the estimation by the Kalman filtering. Estimated road profiles and the real road profile (left). PSD of different estimations and the real road profile (right)

When comparing Figs 8 and 9, it can be noticed that the ICA is robust to the variation of the vehicle speed, but in the Kalman filtering there is a loss of estimation which is especially 
noticeable in the frequency spectrum. This will affect the vehicle responses when driving at different speeds (Fauriat et al., 2016).

\section{Conclusions}

Based on the obtained results, it appears that the ICA is an appropriate technique that enables one to gather all the road profile information even in the presence of payload or speed variations. This proposed method provides a good estimation both in time and frequency domain. The validity of this method has been demonstrated through comparison with the augmented Kalman filtering technique which has some limitations.

The strength of the ICA technique is that, apart from the fact that it is applicable with no need to specific road instruments, it is also inexpensive. Moreover, unlike the Kalman filtering which needs more than three vehicle responses, this method just needs one accelerometer to gather the sprung mass acceleration, and so it can resolve the inverse problem. The ICA has a great potential to identify the road disturbance. Therefore, it can be used over thousands of kilometers as a real time estimation which is inexpensive and fast enough.

In the future work, the proposed method ICA will be coupled with active control to detect in real time road deformation and, then, intuitively adapt the active suspension even before the car reaches the excitation so that the passenger comfort could be achieved.

Acknowledgments

Special thanks go to Vicente Zarzoso and Pierre Comon for making the ICA algorithm (Zarzoso and Comon, 2010).

\section{References}

1. Abbes M.S., Chanbane M.M., Akrout A., Fakhfakh T., Haddar M., 2011, Vibratory behavior of a double panel system by the operational modal analysis, International Journal of Modeling, Simulation, and Scientific Computing, 2, 459-479

2. Agharkakli A., Sabet G.S., Barouz A., 2012, Simulation and analysis of passive and active suspension system using quarter car model for different road profile, International Journal of Engineering Trends and Technology, 3, 636-644

3. Akrout A., Tounsi D., Taktak M., Abbès M.S., Haddar M., 2012, Estimation of dynamic system's excitation forces by the independent component analysis, International Journal of Applied Mechanics, 4, 1250032

4. Basha S.I.A., RaO N.A.N., 2012, Dynamic analysis of MacPherson suspension system by using FE method, International eJournal of Mathematics and Engineering, 155, 1423-1433

5. Bayrakdar Ö., 2010, Random vibration of a road vehicle, Master diploma, Graduate School of Engineering and Sciences of Izmir Institute of Technology, Izmir, July

6. Ben Hassen D., Et Al., 2017a, Application of the independent component analysis in the estimation of the road disturbance applied on a nonlinear suspension system, International Conference Design and Modeling of Mechanical Systems, Springer, Cham, 281-289

7. Ben Hassen D., Et AL., 2017b, Application of the operational modal analysis using the independent component analysis for a quarter car vehicle model, Advances in Acoustics and Vibration, Springer, Cham, 125-133

8. Ben HASSEN D., ET AL., 2017c, Estimation of road disturbance for a non linear half car model using the independent component analysis, Signal Processing Applied to Rotating Machinery Diagnostics (SIGPROMD’2017), Springer, Cham, 96-103 
9. Ben Hassen D., ET AL., 2017d, Road profile estimation using the dynamic responses of the full vehicle model, Applied Acoustics

10. Comon P., 1994, Independent component analysis, a new concept? Signal Processing, 36, 287-314

11. Fang Z., Shu W., Du D., Xiang B., He Q., He K., 2011, Semi-active suspension of a full-vehicle model based on double-loop control, Procedia Engineering, 16, 428-437

12. Fauriat W., Mattrand C., Gayton N., Beakou A., Cembrzynski T., 2016, Estimation of road profile variability from measured vehicle responses, Vehicle System Dynamics, 54, 5, 585-605

13. Gelle G., Colas M., Serviere C., 2003, Blind source separation: A new pre-processing tool for rotating machines monitoring? IEEE Transactions on Instrumentation and Measurement, 52, 790-795

14. Gopala Rao L.V.V., Narayanan S., 2008, Control of response of a quarter-car vehicle model with optimal skyhook damper, International Journal of Vehicle Autonomous Systems, 6, 396-418

15. Harris N.K., González A., O’Brien E.J., McGetrick P., 2010, Characterisation of pavement profile heights using accelerometer readings and a combinatorial optimisation technique, Journal of Sound and Vibration, 329, 497-508

16. Hunt H.E.M., 1991, Stochastic modelling of traffic-induced ground vibration, Journal of Sound and Vibration, 144, 53-70

17. Hyvärinen A., OjA E., 2000, Independent component analysis: algorithms and applications, Neural Networks, 13, 411-430

18. ISO, 8608, 1995, Mechanical VibrationRoad Surface ProfilesReporting of Measured Data

19. Jutten C., Herault J., 1991, Blind separation of sources. Part I: An adaptive algorithm based on neuromimetic architecture, Signal Processing, 24, 1-10

20. Kalman R.E., 1960, A new approach to linear filtering and prediction problems, Transactions of the ASME - Journal of Basic Engineering, Series D, 82, 35-45

21. Karnopp D., 1983, Active damping in road vehicle suspension systems, Vehicle System Dynamics, 12, 291-311

22. Karnopp D., Heess G., 1991, Electronically controllable vehicle suspensions, Vehicle System Dynamics, 20, 207-217

23. Kim H.J., YAng H.S., PARK Y.P., 2002, Improving the vehicle performance with active suspension using road-sensing algorithm, Computers and Structures, 80, 1569-1577

24. Li P., LAm J., Cheung K.C., 2014, Multi-objective control for active vehicle suspension with wheelbase preview, Journal of Sound and Vibration, 333, 5269-5282

25. Nakamura H., Nakano K., Oohashi R., Hu H., Uchiyama Y., Kakihara S., 2012, Road profile estimation from car body vibration using independent component analysis, ASME 20125th Annual Dynamic Systems and Control Conference Joint with the JSME 201211 th Motion and Vibration Conference

26. Purushotham A., 2013, Comparative simulation studies on MacPherson suspension system, International Journal of Modern Engineering Research (IJMER), 3, 1377-1381

27. Savaresi S.M., Spelta C., 2009, A single-sensor control strategy for semi-active suspensions, IEEE Transactions on Control Systems Technology, 17, 143-152

28. SikA G., 2008, Dynamique des transmissions en régime transitoire, PhD Thesis, Ecole Nationale Supérieure de Mécanique et de Microtechnique de Besançon, France

29. Solmmirzaei A., Azadi S., Kazemi R., 2012, Road profile estimation using wavelet neural network and 7-DOF vehicle dynamic systems, Journal of Mechanical Science and Technology, 26, 3029-3036 
30. Taktak M., Tounsi D., Akrout A., Abbès M.S., Haddar M., 2012, One stage spur gear transmission crankcase diagnosis using the independent components method, International Journal of Vehicle Noise and Vibration, 8, 387-400

31. Unger A., Schimmack F., Lohmann B., Schwarz R., 2013, Application of LQ-based semi-active suspension control in a vehicle, Control Engineering Practice, 21, 1841-1850

32. Welch G., Bishop G., 2006, An Introduction to the Kalman Filter, Department of Computer Science, University of North California at Chapel Hill

33. YAN Z., 2012, Road condition predicting with Kalman filter for magneto-rheological damper in suspension system, Thesis, Department of Applied Signal Processing, Blekinge Institute of Technology, July

34. Zarzoso V., Comon P., 2010, Robust independent component analysis by iterative maximization of the kurtosis contrast with algebraic optimal step size, IEEE Transactions on Neural Networks, 21, 248-261

Manuscript received July 13, 2018; accepted for print December 13, 2018 